\section{LA OBESIDAD EN EL PERÚ: RECOMENDACIONES PARA UN SISTEMA DE VIGILANCIA}

\section{OBESITY IN PERU: RECOMMENDATIONS FOR A SURVEILLANCE SYSTEM}

\author{
Julio A. Poterico ${ }^{1, a}$, Rodrigo M. Carrillo-Larco ${ }^{2, b}$, \\ Renato Quispe ${ }^{3, \text { a }}$
}

Sr. Editor, las enfermedades crónicas no transmisibles (ENT) constituyen la principal carga de enfermedad en el mundo, que afecta más a países de medianos y bajos ingresos. En la actualidad, las organizaciones internacionales exhortan la vigilancia de los factores de riesgo comunes a las ENT: la inactividad física, patrones de alimentación, y consumo de tabaco y alcohol (1). El sobrepeso y la obesidad también forman parte de los pilares de la vigilancia de las ENT. La Organización Mundial de la Salud (OMS) sugiere el uso del índice de masa corporal (IMC) como un indicador de malnutrición en adultos, así como otras referencias antropométricas para el caso de los niños y adolescentes (1).

En el Perú, se cuenta con un sistema de vigilancia de salud en las mujeres en edad fértil y niños menores de 5 años mediante la Encuesta Nacional Demográfica y de Salud Familiar (ENDES). Esta última ha venido presentando información orientada hacia las enfermedades infecciosas, desnutrición infantil y acceso a servicios de salud; aunque recientemente (desde el 2010 y de manera progresiva) ha incorporado recolección de algunos datos de ENT. Sin embargo, la exclusión del sexo masculino y otros grupos de edad en la ENDES conlleva a un vacío en la supervisión de la obesidad en la población peruana. Así por ejemplo, un estudio reciente -con información de la Encuesta Nacional de Hogares (ENAHO) en los años 2009 y 2010-demostró una distribución diferente de los determinantes sociales en el exceso de peso según la edad, sexo y nivel socioeconómico en el Perú ${ }^{(2)}$. Esto demuestra la necesidad de un sistema constante y completo en la vigilancia de la obesidad en una sociedad con rápida transición nutricional, como la peruana.

El sistema de vigilancia de sobrepeso y obesidad en los países de bajos y medianos ingresos se

\footnotetext{
CRONICAS Centro de Excelencia en Enfermedades Crónicas, Universidad Peruana Cayetano Heredia. Lima, Perú.

2 Facultad de Medicina "Alberto Hurtado", Universidad Peruana Cayetano Heredia. Lima, Perú.

3 Instituto de Medicina Tropical "Alexander von Humboldt", Universidad Peruana Cayetano Heredia. Lima, Perú.

a Médico cirujano, ${ }^{\text {b }}$ estudiante de Medicina

Recibido: 14-10-12 Aprobado: 31-10-12
}

Citar como: Poterico JA, Carrillo-Larco RM, Quispe R. La obesidad en el Perú: recomendaciones para un sistema de vigilancia. Rev Peru Med Exp Salud Publica. 2012;29(4):581. ha estado desarrollando a través del IMC (3). Sin embargo, este indicador tiene como limitación su baja sensibilidad para identificar a la población con exceso de grasa corporal ${ }^{(4)}$. Esto sugiere que las personas clasificadas como obesas por el porcentaje de grasa corporal, evidencian factores de riesgo cardiovascular, aun cuando el IMC se encuentre en rangos normales o en sobrepeso. El radio cintura-altura, el radio cinturacadera y la circunferencia abdominal, estimarían mejor (en comparación con el IMC) el riesgo de tener alguna ENT (5).

En consecuencia, recomendamos la extensión y complementación de las encuestas nacionales, abarcando tanto a la población masculina como la femenina, y todos los grupos etarios de una manera constante; además de incluir medidas antropométricas adicionales al IMC. Ello asistiría en la generación de políticas públicas con evidencia para respaldar estrategias efectivas contra la pandemia de la obesidad.

Agradecimientos: al Dr. J. Jaime Miranda por los comentarios realizados en la elaboración de la presente publicación.

Fuente de financiamiento: autofinanciado.

Conflictos de Interés: los autores declaran no tener conflicto de interés en la publicación de este artículo.

\section{REFERENCIAS BIBLIOGRÁFICAS}

1. World Health Organization. A comprehensive global monitoring framework including indicators and a set of voluntary global targets for the prevention and control of noncommunicable diseases. Geneva: World Health Organization; 2012.

2. Álvarez-Dongo D, Sánchez-Abanto J, Gómez-Guizado G, Tarqui-Mamani C. Sobrepeso y obesidad: Prevalencia y determinantes sociales del exceso de peso en la población peruana. Rev Peru Med Exp Salud Publica. 2012;29(3):303-13.

3. Alwan A, MacLean DR, Riley ML, d'Espaignet ET, Mather $\mathrm{CD}$, Stevens GA, et al. Monitoring and surveillance of chronic non-communicable diseases: progress and capacity in highburden countries. Lancet. 2010;376(9755):1861-8.

4. Okorodudu DO, Jumean MF, Montori VM, Romero-Corral A, Somers VK, Erwin PJ, et al. Diagnostic performance of body mass index to identify obesity as defined by body adiposity: a systematic review and meta-analysis. Int J Obes (Lond). 2010;34(5):791-9.

5. Herrera VM, Casas JP, Miranda JJ, Perel P, Pichardo R, Gonzalez A, et al. Interethnic differences in the accuracy of anthropometric indicators of obesity in screening for high risk of coronary heart disease. Int J Obes (Lond). 2009;33(5):568-76.

Correspondencia: Julio Antonio Poterico Rojas

Dirección: Av. Armendáriz 497, 2 do piso, Miraflores, Lima 18 Perú. Teléfono: (511) 2416978

Correo electrónico:julio.poterico@upch.pe 